\title{
Deriving Behavior of Boolean Bioregulatory Networks from Subnetwork Dynamics
}

\author{
Heike Siebert
}

\begin{abstract}
In the well-known discrete modeling framework developed by $\mathrm{R}$. Thomas, the structure of a biological regulatory network is captured in an interaction graph, which, together with a set of Boolean parameters, gives rise to a state transition graph describing all possible dynamical behaviors. For complex networks the analysis of the dynamics becomes more and more difficult, and efficient methods to carry out the analysis are needed. In this paper, we focus on identifying subnetworks of the system that govern the behavior of the system as a whole. We present methods to derive trajectories and attractors of the network from the dynamics suitable subnetworks display in isolation. In addition, we use these ideas to link the existence of certain structural motifs, namely circuits, in the interaction graph to the character and number of attractors in the state transition graph, generalizing and refining results presented in [10]. Lastly, we show for a specific class of networks that all possible asymptotic behaviors of networks in that class can be derived from the dynamics of easily identifiable subnetworks.
\end{abstract}

\section{Introduction}

When modeling biological systems, one first has to decide what kind of modeling framework is best suited to incorporate the available data and to yield results without too many additional assumptions about the system. If only coarse information is available, as is often the case when studying biological regulatory networks, logical modeling approaches lend themselves well to capturing the essential, qualitative features of the system. In the 70's, R. Thomas introduced a discrete modeling formalism, which has been continuously further developed and successfully applied to biological problems (see [13], [14] and references therein). The structure of the network is captured in a directed, signed graph called interaction graph. Edges represent activating or inhibiting interactions between components, which in turn 
are represented by Boolean variables. A component is considered active if the associated variable has value 1 and inactive otherwise. Boolean parameter values specify a function that determines the dynamical behavior. Biologically realistic rules are employed to derive a state transition graph from the Boolean function, which amounts to a non-deterministic representation of all possible behaviors of the system.

Since the representation of the dynamics is in some sense comprehensive, the analysis of the behavior is rather involved for complex networks. One idea to simplify the analysis is to deconstruct the complex network into simpler building blocks, to analyze their dynamics in isolation and then derive information about the network dynamics from the subnetwork behavior. Clearly, this method will not work for arbitrarily chosen subnetworks, because further components and interactions influence its behavior once it is embedded in a complex system. If, however, we can identify subnetworks that somehow govern the behavior of the larger network, at least in some part of state space, then we can infer useful results about the whole network from looking at those subnetworks.

We approach the problem of finding suitable subnetworks in the following way. We basically look for parts of state space, where some of the components of the network remain stable independent of the values of other network components. A notion that formalizes this idea is the notion of singular steady state. It was first introduced by R. Thomas and E.H. Snoussi in [11] for a certain class of multivalued discrete functions. Here, a so-called singular value represent the threshold of an interaction, which allows a refined representation of the network dynamics. We adapted these ideas to a Boolean setting in [10], resulting in definitions and results for a restricted class of Boolean models. In this paper, we substantially generalize the framework introduced in [10]. We allow characteristics, i. e., the sign of network interactions to depend on the current state of the system. Whether a component has an activating or inhibiting influence on its target may depend on the activity of certain cofactors. A well-known example is the DNA-binding protein TCF which can repress as well as activate the same target genes. TCF acts as activator in the presence of $\beta$-catenin, induced by WNT signaling, while the co-expression of the protein TLE converts TCF into a repressor. We call systems including such ambiguous interactions context sensitive. Adaptations in the definition of interaction graphs and parameters allow us to include context sensitive systems in our considerations.

In this setting, a component can adopt the singular value $\theta$ in addition to the two regular values 1 and 0 . Again, the singular value can be interpreted as the threshold value of an interaction, and thus represents a state where we do not know whether or not the corresponding interaction is active. Considering such states can help us to a clearer understanding of the component dependencies in the system. To obtain a refined representation of the structure of the system with respect to its dynamics, we exploit the concept of local interactions graphs. It was already successfully used in [5] and [4], and allows for a better understanding of what structures in the interaction graph influence the system's behavior in a given 
state. Combining these ideas, we introduce local interaction graphs of singular steady states which allow us to identify subnetworks that govern the behavior of the whole system.

The paper is organized as follows. In Sect. 2 we introduce the Boolean framework we use to describe regulatory networks. The following section clarifies the relation between the function $f$ governing the system's dynamics and the structure of the interaction graph, which leads to the notion of local interaction graph. Subsequently, we introduce singular steady states. In Sect. 5, we employ the concept of local interaction graphs for singular steady states. We identify subnetworks that govern the system's behavior, and introduce a procedure to derive the dynamics and in particular attractors of the network from the corresponding subnetwork dynamics. We also focus on subnetworks that basically consist of an isolated circuit, and link there existence in the interaction graph to certain dynamical characteristics of the network. In Sect. 6, we then analyze a specific class of networks the properties of which allow us to easily find subnetworks from which we can derive all possible attractors of the original system. We end the paper with concluding remarks and perspectives for future work.

This is the extended version of a paper presented at the Algebraic Biology conference 2008 [9].

\section{Regulatory Networks}

As already mentioned, a directed, signed graph is used in the Thomas formalism to capture the network structure of a regulatory system. We are now interested in a more general representation that allows for the interaction sign to depend on the current state of the system. To accurately describe the structure of such context sensitive networks we use directed multigraphs that allow for parallel edges. Multigraphs have been used in a similar way in $[2]$. We set $\mathcal{B}:=\{0,1\}$.

Definition 2.1. An interaction (multi-)graph (or bioregulatory (multi-)graph) $\mathcal{I}$ is a labeled directed multigraph with vertex set $V:=\left\{\alpha_{1}, \ldots, \alpha_{n}\right\}, n \in \mathbb{N}$, and edge set $E \subseteq V \times V \times\{+,-\}$.

The vertices $\alpha_{1}, \ldots, \alpha_{n}$ represent the components of the regulatory network such as genes, RNA, or proteins. We view each component $\alpha_{i}$ as a variable that adopts values in $\mathcal{B}$. The value 1 signifies that the component is active, i. e., it influences its interaction targets according to the interaction signs. For example, if some substance concentration needs to cross a threshold in order to influence some target component, then the corresponding Boolean value is 0 as long as the concentration is below, and 1 if the concentration is above the threshold.

When analyzing the interaction graph of a network we are interested in certain structural motives. We focus on so-called (feedback) circuits. Here, a circuit is a tuple $\left(e_{1}, \ldots, e_{r}\right)$ of edges $e_{i}=\left(k^{i}, l^{i}, \varepsilon\right) \in E$ such that all $k^{i}, i \in\{1, \ldots, r\}$, are pairwise distinct, and $l^{i}=k^{i+1}$ for all $i \in\{1, \ldots, r\}$ modulo $r$. The sign of a circuit is the product of the signs of its edges. Note that in a multigraph a 
circuit is not uniquely determined by its vertices. Figure 1 shows an interaction graph with two circuits consisting of the vertices $\alpha_{2}$ and $\alpha_{3}$ : the positive circuit $\left(\left(\alpha_{2}, \alpha_{3},+\right),\left(\alpha_{3}, \alpha_{2},+\right)\right)$ and the negative circuit $\left(\left(\alpha_{2}, \alpha_{3},+\right),\left(\alpha_{3}, \alpha_{2},-\right)\right)$.

To simplify notation, we identify each vertex $\alpha_{i}$ with its index $i$, and denote $e_{i j}^{\varepsilon}:=(i, j, \varepsilon)$ for all $(i, j, \varepsilon) \in E$. For each $\alpha_{i}$ we denote by $\operatorname{Pred}\left(\alpha_{i}\right)$ the set of predecessors of $\alpha_{i}$, i. e., the set of vertices $\alpha_{j}$ such that there is an edge $\left(\alpha_{j}, \alpha_{i}, \varepsilon\right)$ for some $\varepsilon \in\{+,-\}$ in $E$. To identify parallel edges we set $E^{\prime \prime}=\left\{(i, j) \mid \exists e_{i, j}^{\varepsilon}, e_{i, j}^{\varepsilon^{\prime}} \in\right.$ $\left.E: \varepsilon \neq \varepsilon^{\prime}\right\}$ and $E^{\prime}=E \backslash E^{\prime \prime}$.

An interaction graph holds no information about dynamical behavior. Next we give a formal definition of the term bioregulatory network that includes information on structure as well as dynamics. The notation is based on ideas introduced in [1] and [7].

Definition 2.2. Let $\mathcal{I}=(V, E)$ be an interaction graph comprising $n$ vertices. A state of the system described by $\mathcal{I}$ is a tuple $s \in \mathcal{B}^{n}$. The set of (regular) resource edges $R_{j}(s)=R_{j}^{\mathcal{I}}(s)$ of $\alpha_{j}$ in state $s=\left(s_{1}, \ldots, s_{n}\right)$ is the set

$$
\left\{\left(\alpha_{i}, \alpha_{j}, \varepsilon\right) \in E \mid\left(\varepsilon=+\wedge s_{i}=1\right) \vee\left(\varepsilon=-\wedge s_{i}=0\right)\right\} .
$$

Given a set

$$
K(\mathcal{I}):=\left\{K_{j, R_{j}(s)} \mid j \in\{1, \ldots, n\}, s \in \mathcal{B}^{n}\right\}
$$

of (logical) parameters, which adopt values in $\mathcal{B}$, we define the Boolean function $f=f^{K(\mathcal{I})}: \mathcal{B}^{n} \rightarrow \mathcal{B}^{n}, s \mapsto\left(K_{1, R_{1}(s)}, \ldots, K_{n, R_{n}(s)}\right)$. The pair $N:=(\mathcal{I}, f)$ is called bioregulatory network.

The behavior of a component $\alpha_{j}$ is determined by the influences its predecessors exert on it. The set of resource edges $R_{j}(s)$ contains all edges that contribute to an activation of $\alpha_{j}$ in state $s$. Note that here the absence of an inhibiting influence (represented by a negative edge) is interpreted as an activating influence on the target component. With this interpretation we have that for every $s \in \mathcal{B}^{n}$ there is $\varepsilon \in\{+,-\}$ such that $e_{i j}^{\varepsilon} \in R_{j}(s)$, if $(i, j) \in E^{\prime \prime}$. If $(i, j) \in E^{\prime}$, then $R_{j}(s)$ may or may not contain the corresponding edge $e_{i, j}^{\varepsilon}$, depending on $s$.

In Fig. 1 an interaction graph and a choice of parameter values are given. For $\alpha_{1}$ and $\alpha_{3}$ the parameters depend on whether or not the single positive edge ending in $\alpha_{1}$ resp. $\alpha_{3}$ is effective or ineffective. We have $R_{1}(s)=\emptyset$ for all states $s$ with $s_{1}=0$, and $R_{1}(s)=\left\{e_{11}^{+}\right\}$for all $s$ with $s_{1}=1$, while the resource edge sets for $\alpha_{3}$ depend similarly on $e_{23}^{+}$. The component $\alpha_{2}$ is influenced by both $\alpha_{1}$ and $\alpha_{3}$ via two parallel edges, respectively. Thus the set of resources is never empty. For example, we have $R_{2}((0,0,1))=\left\{e_{12}^{-}, e_{32}^{+}\right\}$. A closer look at the choice of parameter values allows the following interpretation. If $\alpha_{1}$ has activity level 0 , then the influence of $\alpha_{3}$ on $\alpha_{2}$ corresponds to an activating influence: if $\alpha_{3}$ is inactive, $\alpha_{2}$ tends to inactivity represented by the parameter $K_{2,\left\{e_{12}^{-}, e_{32}^{-}\right\}}=0$, and if $\alpha_{3}$ is active $\alpha_{2}$ tends to activity since $K_{2,\left\{e_{12}^{-}, e_{32}^{+}\right\}}=1$. If $\alpha_{1}$ has value 1 , then the situation is reversed and $\alpha_{3}$ inhibits $\alpha_{2}$. The system is context sensitive. 


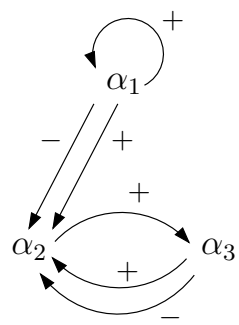

$$
\begin{array}{ll}
K_{1, \emptyset} & =0 \\
K_{1,\left\{e_{11}^{+}\right\}} & =1 \\
K_{2,\left\{e_{12}^{+}, e_{32}^{+}\right\}} & =0 \\
K_{2,\left\{e_{12}^{+}, e_{32}^{-}\right\}} & =1 \\
K_{2,\left\{e_{12}^{-}, e_{32}^{+}\right\}} & =1 \\
K_{2,\left\{e_{12}^{-}, e_{32}^{-}\right\}} & =0 \\
K_{3, \emptyset} & =0 \\
K_{3,\left\{e_{23}^{+}\right\}} & =
\end{array}
$$

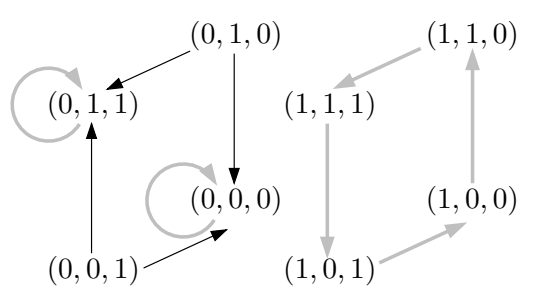

FIGURE 1. Interaction graph of a system comprising three components, a list of all parameters with an assignment of Boolean values, and the corresponding state transition graph. The heavier gray edges indicate attractors.

In [10], the parameters correspond to sets of resource vertices, i. e., the influence of one component on another cannot change depending on the current state of the system. The network shown in Fig. 1 cannot be represented with that restriction. However, the notion of resource edges and resource vertices are equivalent, if there are no parallel edges in the interaction graph.

The parameters determine the behavior of the system as follows. The Boolean value of the parameter $K_{j, R_{j}(s)}$ indicates how the activity level, i. e., the value of the component $\alpha_{j}$ will evolve from its value in state $s$. It will increase (resp. decrease) if the parameter value is greater (resp. smaller) than $s_{i}$. The activity level stays the same if both values are equal. Thus, the function $f$ maps a state $s$ to the state the system tends to evolve to. However, if a state and its image differ in more than one component, we take the following consideration into account. In a biological system two different processes of change in activity level represented by the value change of two distinct components will not take the exact same amount of time. Thus we assume that in the discrete dynamical representation a state differs from its successor in at most one component. This procedure is called asynchronous update in Thomas' framework. By applying this idea we derive a non-deterministic representation of the dynamics which we again formalize as a directed graph.

Definition 2.3. The state transition graph $\mathcal{S}_{N}$ describing the dynamics of the network $N$ is a directed graph with vertex set $\mathcal{B}^{n}$. For states $s=\left(s_{1}, \ldots, s_{n}\right)$ and $s^{\prime}=\left(s_{1}^{\prime}, \ldots, s_{n}^{\prime}\right)$, there is an edge $s \rightarrow s^{\prime}$ if and only if $s^{\prime}=f(s)=s$ or $s_{i}^{\prime}=f_{i}(s)$ for some $i \in\{1, \ldots, n\}$ satisfying $s_{i} \neq f_{i}(s)$ and $s_{j}^{\prime}=s_{j}$ for all $j \neq i$.

On the right in Fig. 1 we see the state transition graph corresponding to the given interaction graph and parameters. The dynamics are non-deterministic. For example, there are two edges leaving the state $(0,1,0)$, representing two different behaviors of the system. 
Every possible behavior of the system is captured in the corresponding state transition graph. To analyze the graph we use, in addition to standard terminology from graph theory such as paths and cycles, the following concepts.

Definition 2.4. An infinite path $\left(s_{0}, s_{1}, \ldots\right)$ in $\mathcal{S}_{N}$ is called trajectory. A nonempty set of states $D$ is called trap set if every trajectory starting in $D$ never leaves $D$. A trap set $A$ is called attractor if for all $s^{1}, s^{2} \in A$ there is a path from $s^{1}$ to $s^{2}$ in $\mathcal{S}_{N}$. A cycle $C:=\left(s^{1}, \ldots, s^{r}, s^{1}\right), r \geq 2$, is called a trap cycle if every $s^{j}$, $j \in\{1, \ldots, r\}$, has only one outgoing edge in $\mathcal{S}_{N}$, i. e., the trajectory starting in $s^{1}$ is unique. A state $s$ is called steady state, if there exists an edge $s \rightarrow s$, i. e. if $f(s)=s$.

In other words, the attractors correspond to the terminal strongly connected components of the graph. Steady states as well as trap cycles are attractors. The attractors in the state transition graph given in Fig. 1 are the sets containing the steady states, i.e., $\{(0,0,0)\}$ and $\{(0,1,1)\}$, and the set containing the states of the trap cycle in the graph, i. e., $\{(1,0,0),(1,1,0),(1,1,1),(1,0,0)\}$.

The behavior of a system becomes, at least to some degree, predictable and stable inside an attractor. Often, a sensible biological interpretation can be found for an attractor. In cell differentiation, the different stable states reached at the end of development may be represented by distinct steady states in the state transition graph. Attractors of cardinality greater than one imply cyclic behavior, and thus can often be identified with homeostasis of sustained oscillatory activity, as can be found in the cell cycle or circadian rhythm.

State transition graphs always contain at least one attractor. The proof of the following more precise statement can be found in [10].

Proposition 2.5. For every state $s \in \mathcal{B}^{n}$ exists a trajectory in $\mathcal{S}_{N}$ which starts in $s$ and leads to an attractor.

Coming to the end of this section, we note a useful observation. If some vertex $\alpha_{i}$ in $\mathcal{I}$ does not have a predecessor, then clearly $a_{i}=K_{i, \emptyset}$ for every state $a=\left(a_{1}, \ldots, a_{n}\right)$ in an attractor. Similarly, we know the values $a_{j}$ for vertices the only predecessor of which is $\alpha_{i}$, and so on. That is, we can easily determine the dynamical behavior of such vertices, which leads to the same fixed values of those components for every initial state of the system. Throughout the remainder of the paper we exclude such components and assume that every vertex in $\mathcal{I}$ has a predecessor. We still allow the system to have input values in the sense of components maintaining their current activity level independent of the values of the other components. Such an input component is represented as a vertex with its only incoming edge being a positive self-loop.

\section{Functionality and Local Interaction Graphs}

Throughout the paper let $N:=\left(\mathcal{I}=(V, E), f=f^{K(\mathcal{I})}\right)$ be a bioregulatory network comprising $n$ components. When analyzing the system, it is an interesting 
question whether it is possible to link structural network characteristics to dynamical characteristics. In order to obtain sensible results, however, we need to make sure that the structure of the network captured in the interaction graph and the rules governing the behavior of the system represented by the Boolean function $f$ do not contradict each other. That is, the choice of parameter values should be consistent with the information inherent in the interaction graph. We require that each edge represented in the interaction graph should have a notable effect on the system's dynamics. Moreover, the edge's character given by its sign should be reflected in its dynamical impact.

To formalize these requirements we introduce the following notation. Recall that we interpret the absence of inhibition as a potentially activating effect. So, if we deal with parallel edges from a vertex $i$ to a vertex $j$, that is if $(i, j) \in E^{\prime \prime}$, we know that for every $s \in \mathcal{B}^{n}$ there is $\varepsilon \in\{+,-\}$ such that $e_{i j}^{\varepsilon} \in R_{j}(s)$. If there is only a single edge, i.e. $(i, j) \in E^{\prime}$, then $R_{j}(s)$ may or may not contain the corresponding edge $e_{i, j}^{\varepsilon}$, depending on $s$.

For $j \in\{1, \ldots, n\}$, set $M_{j}^{\mathcal{I}}:=M_{j}:=\left\{R_{j}(s) \mid s \in \mathcal{B}^{n}\right\}$. Then, by the above considerations, each $M \in M_{j}$ can be written as $M=\bigcup_{i \in \operatorname{Pred}(j)} L_{i}$ with $L_{i}=\left\{e_{i, j}^{\varepsilon}\right\}$ for some $\varepsilon \in\{+,-\}$, if $(i, j) \in E^{\prime \prime}$, and $L_{i}=\emptyset$ or $L_{i}=\left\{e_{i, j}^{\varepsilon}\right\} \subset E$, if $(i, j) \in E^{\prime}$. By definition we have $K(\mathcal{I})=\left\{K_{j, M} \mid j \in\{1, \ldots, n\}, M \in M_{j}\right\}$.

We want to ensure that the choice of parameter values does not contradict the information inherent in the interaction graph. As mentioned above, that means we have to check for the existence and the character, positive or negative, of influence on the system dynamics exhibited by each interaction. To do so, we again have to distinguish between edges in $E^{\prime}$ and $E^{\prime \prime}$. For $e=(i, j, \varepsilon),(i, j) \in E^{\prime}$, we have $M \cup\{e\} \in M_{j}$ for all $M \in M_{j}$, and we demand that $K_{j, M} \leq K_{j, M \cup\{e\}}$ for all $M \in M_{j}$. Recall that the addition of an edge to the set of resources always signifies increasing activating influence. So, the condition ensures that increasing activating influence does not result in a decrease of component activity level. To ensure that $e$, at least for some state, has a notable impact on the dynamics, we extend the condition and get:

$$
\forall M \in M_{j}: K_{j, M} \leq K_{j, M \cup\{e\}} \quad \text { and } \quad \exists M^{\prime} \in M_{j}: K_{j, M^{\prime}}<K_{j, M^{\prime} \cup\{e\}} .
$$

In the case $(i, j) \in E^{\prime \prime}$, there exists $e^{\prime}=\left(i, j, \varepsilon^{\prime}\right)$ with $\varepsilon \neq \varepsilon^{\prime}$. Since $\alpha_{i}$ influences $\alpha_{j}$ positively as well as negatively depending on the current state, we cannot impose a general monotonicity condition on the parameters as in the first part of (3.1). However, again we require that there is at least one state where the addition of $e$ to the set of resources induces an increase in the parameter value. Otherwise the edge $e$ would be superfluous. Since in every given state either $e$ or $e^{\prime}$ is contained in the set of resources, we compare parameter values for sets $M \in M_{j}$ and $\left(M \backslash\left\{e^{\prime}\right\}\right) \cup\{e\}$. We obtain the condition

$$
\exists M^{\prime} \in M_{j}: K_{j, M^{\prime}}<K_{j,\left(M^{\prime} \backslash\left\{e^{\prime}\right\}\right) \cup\{e\}} \cdot
$$

We call edges that satisfy condition (3.1) resp. (3.2) functional. This concept of functionality is an adaptation of the notion of functionality introduced in [10]. 
In the following, we always assume that all edges in the interaction graph are functional.

We have already seen in Sect. 2 that for the example in Fig. $1 R_{1}(s)=\emptyset$ for all states $s$ with $s_{1}=0$, and $R_{1}(s)=\left\{e_{11}^{+}\right\}$for all $s$ with $s_{1}=1$. Thus $M_{1}=\left\{\emptyset,\left\{e_{11}^{+}\right\}\right\}$. Similarly $M_{3}=\left\{\emptyset,\left\{e_{23}^{+}\right\}\right\}$. The choice of Boolean values for the parameters satisfies condition (3.1) and ensures the functionality of the edges $e_{11}^{+}$ and $e_{23}^{+}$. Since $\alpha_{2}$ is influenced by both $\alpha_{1}$ and $\alpha_{3}$ via two parallel edges, we get $M_{2}=\left\{\left\{e_{12}^{+}, e_{32}^{+}\right\},\left\{e_{12}^{+}, e_{32}^{-}\right\},\left\{e_{12}^{-}, e_{32}^{+}\right\},\left\{e_{12}^{-}, e_{32}^{-}\right\}\right\}$. Again the choice of parameter values renders all edges functional.

A different choice of parameter values leads to different results. If we set $K_{2,\left\{e_{12}^{+}, e_{32}^{+}\right\}}=K_{2,\left\{e_{12}^{-}, e_{32}^{+}\right\}}=1$ and $K_{2,\left\{e_{12}^{+}, e_{32}^{-}\right\}}=K_{2,\left\{e_{12}^{-}, e_{32}^{-}\right\}}=0$, then verification of conditions (3.1) and (3.2) shows that $e_{12}^{+}, e_{12}^{-}$and $e_{32}^{-}$are not functional. Only the edge $e_{32}^{+}$is functional and influences the system's dynamics.

It now may seem that we put a lot of restrictions on the choice of parameter values and thus on the function $f$ by demanding functionality of all edges. But in fact we only make sure that the graph representation of the system's structure fits the structural information encoded in $f$. This is no restriction on the Boolean function $f$ as the following statement shows. The proof can be found in [9].

Proposition 3.1. Let $g: \mathcal{B}^{n} \rightarrow \mathcal{B}^{n}$ be a Boolean function. Then there exists an interaction graph $\mathcal{I}=(V, E)$ and a set of parameters $K(\mathcal{I})$ such that $g=f^{K(\mathcal{I})}$.

The above statement also illustrates the fact that the interaction graph holds only coarse information on the system. The same interaction graph may give rise to different dynamics depending on the choice of parameter values. However, a more refined understanding of the network structure is possible, if we consider the impact of interactions on the dynamics with respect to the current state of the system.

Since all edges in the interaction graph are functional, we know that each edge has an impact on the dynamics. However, this influence does not have to be effective in the whole state space $\mathcal{B}^{n}$. To capture local structural aspects we introduce the concept of local interaction graphs. It has already been used in [5] and [4] (see also references therein). In the following, we denote with $\bar{s}^{i}$ the state that coincides with $s$ in all components $j \neq i$ and takes the value $1-s_{i}$ in the $i$-th component.

Definition 3.2. Let $\mathcal{I}=(V, E)$ be an interaction graph with parameter set $K(\mathcal{I})$. Let $s=\left(s_{1}, \ldots, s_{n}\right) \in \mathcal{B}^{n}$. Then we denote by $\mathcal{I}(s)$ the graph with vertex set $V$ and edge set $E(s) \subseteq E$. An edge $(i, j, \varepsilon)$ is in $E(s)$ if and only if

$$
K_{j, R_{j}(s)} \neq K_{j, R_{j}\left(\bar{s}^{i}\right)} \wedge \varepsilon=+\Leftrightarrow s_{i}=K_{j, R_{j}(s)} .
$$

We call $\mathcal{I}(s)$ the (local) interaction graph in state $s$.

Clearly, every edge in the local interaction graph $\mathcal{I}(s)$ is also contained in $\mathcal{I}$, since we use the same parameters to characterize the edges. More precisely, $\mathcal{I}$ is the union of all graphs $\mathcal{I}(s), s \in \mathcal{B}^{n}$. We call $\mathcal{I}$ also the global interaction graph. 
(a)

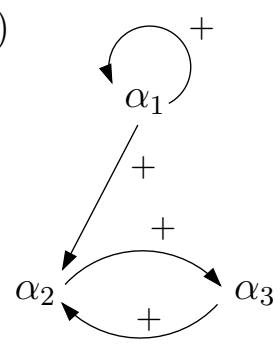

(b)
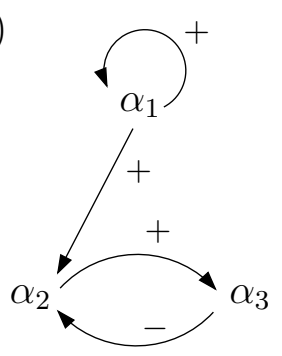

(c)

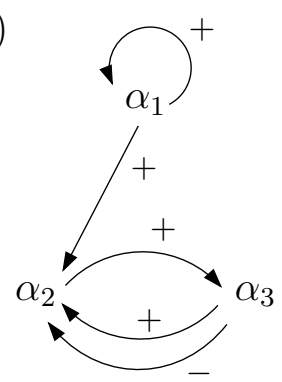

Figure 2. Local interaction graphs corresponding to the graph and parameters given in Fig. 1. $\mathcal{I}((0,0,0))$ in $(\mathrm{a}), \mathcal{I}((1,0,0))$ in (b), $\mathcal{I}((\theta, 0,0))$ in (c).

Note that there are no parallel edges in a local interaction graph. Figure 2(a) and (b) show the graphs $\mathcal{I}((0,0,0))$ and $\mathcal{I}((1,0,0))$ corresponding to the example given in Fig. 1. The local interaction graphs give us a finer understanding of the way the network components interact. They can be seen as a visualization of the discrete Jacobian matrix of the Boolean function $f^{\mathcal{I}}=f$ as introduced in [8], since we have $f_{j}(s)=K_{j, R_{j}(s)}$ for all $s \in \mathcal{B}^{n}$.

\section{Singular States}

In our formalism we only consider whether a component is active or not. We now incorporate a threshold value that allows us to express uncertainty in the sense that we do not know if a certain interaction is effective. We already used this concept in [10] for networks without context sensitivity. Again, we mainly use notation introduced in [7].

Definition 4.1. Set $\mathcal{B}_{\theta}:=\{0, \theta, 1\}$, where $\theta$ is a symbolic representation of the threshold value and satisfies the order $0<\theta<1$. We allow each regulatory component $\alpha_{i}$ to take values in $\mathcal{B}_{\theta}$. The values 0 and 1 are called regular values and $\theta$ is called singular value. The elements of $\mathcal{B}_{\theta}^{n}$ are called states. If all components of a state are regular, it is called regular state, else it is called singular state. For every state $s=\left(s_{1}, \ldots, s_{n}\right)$ we define $J(s):=\left\{i \in\{1, \ldots, n\} \mid s_{i}=\theta\right\}$.

We call $|a, b|$ a qualitative value if $a, b \in \mathcal{B}$ and $a \leq b$. The qualitative value $|0,0|$ is identified with the regular value $0,|1,1|$ with the regular value 1 , and $|0,1|$ with the singular value $\theta$. The relations $\langle$,$\rangle , and =$ are used with respect to this identification.

In the following, we denote $[s]:=\left\{s^{\prime} \in \mathcal{B}^{n} \mid s_{j}^{\prime}=s_{j}\right.$ for all $\left.j \notin J(s)\right\}$ for all $s \in \mathcal{B}_{\theta}^{n}$. The set $[s]$ describes a part of the regular state space which we can be projected on $\mathcal{B}^{k}$, where $k$ is the cardinality of $J(s)$. 
Definition 4.2. We define for all $i \in\{1, \ldots, n\}$

$$
f^{\theta}=f^{K(\mathcal{I}), \theta}: \mathcal{B}_{\theta}^{n} \rightarrow \mathcal{B}_{\theta}^{n} \quad \text { by } \quad f_{i}^{\theta}(s)=\left|K_{i, \min (s)}, K_{i, \max (s)}\right|,
$$

where $K_{i, \min (s)}:=\min \left\{K_{i, R_{i}\left(s^{\prime}\right)} \mid s^{\prime} \in[s]\right\}$ and $K_{i, \max (s)}:=\max \left\{K_{i, R_{i}\left(s^{\prime}\right)} \mid s^{\prime} \in\right.$ $[s]\}$. We call $s \in \mathcal{B}_{\theta}^{n}$ a steady state if $f^{\theta}(s)=s$.

The definition of $K_{i, \min (s)}$ and $K_{i, \max (s)}$ ensures that the image of a regular state under $f^{\theta}$ is again a regular state. More specific, we have $\left.f^{\theta}\right|_{\mathcal{B}^{n}}=f$. If a state has singular components, then $K_{i, \min (s)}$ and $K_{i, \max (s)}$ compare the best and worst case scenario regarding activation for component $i$ by considering all possible combinations of regular values for the singular components of $s$. If the value $f_{i}^{\theta}(s)$ for some $s \in \mathcal{B}_{\theta}^{n}$ is regular, we can deduce that the regular components of $s$ already determine the behavior of the $i$-th component, independent of any predecessors with singular value in $s$. If it is not regular, then we simply do not have enough information to predict what it's future value would be. A singular steady state can then be viewed as a partial steady state of the regular dynamics. We will exploit this fact in the next section.

Thomas and Snoussi already link singular states to circuits in the interaction graph, albeit in a different framework (see [11]). We have adapted their ideas to a Boolean framework without context sensitivity in [10].

Definition 4.3. Let $C=\left(\alpha_{i_{1}}, \ldots, \alpha_{i_{r}}\right)$ be a circuit in $\mathcal{I}$. A state $s=\left(s_{1}, \ldots, s_{n}\right) \in$ $\mathcal{B}_{\theta}^{n}$ is called characteristic state of $C$ if $s_{i_{l}}=\theta$ for all $l \in\{1, \ldots, r\}$.

In general, a characteristic state of a circuit is not unique. The state $(\theta, \ldots, \theta)$ is characteristic for every circuit in $\mathcal{I}$. A simple modification of the reasoning in [10] leads to the following statement.

Theorem 4.4. Every singular steady state is characteristic of some circuit in $\mathcal{I}$.

A singular steady state $s$ can be characterized using only regular states and the function $f$. The idea is to check component-wise the behavior for regular states $s^{+}$and $s^{-}$that satisfy $K_{i, R_{i}\left(s^{+}\right)}=K_{i, \max (s)}$ and $K_{i, R_{i}\left(s^{-}\right)}=K_{i, \min (s)}$ for some $i \in\{1, \ldots, n\}$. The proofs for networks that are not context sensitive are given in [10] and can be easily adapted.

\section{Subnetworks Governing Network Behavior}

In this section we try to find subnetworks of $N$ that in some sense govern the behavior of the whole system, at least in some part of state space. The key idea in this endeavor is to have a closer look at the structural as well as dynamical information inherent in a given singular steady state. As a first step, we adapt the concept of local interaction graphs to singular states. Recall that $J(s)$ is the set of all singular components of a state $s \in \mathcal{B}_{\theta}^{n}$. 
(a)

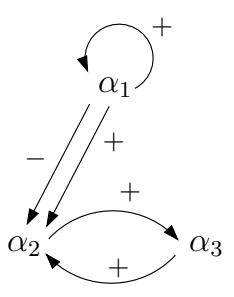

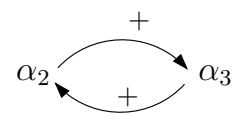

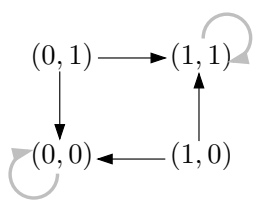

(b)
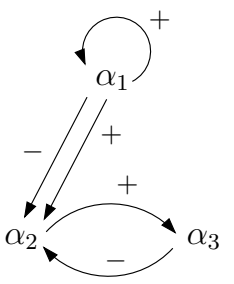
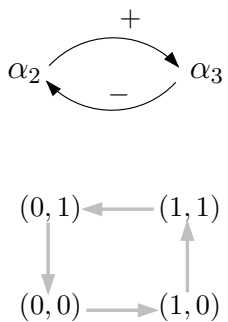

FiguRE 3. Local interaction graph $\mathcal{I}((0, \theta, \theta))$ on the left, $\mathcal{I}^{\theta}((0, \theta, \theta))$ and corresponding state transition graph on the right of (a). Local interaction graph $\mathcal{I}((1, \theta, \theta))$ on the left, $\mathcal{I}^{\theta}((1, \theta, \theta))$ and corresponding state transition graph on the right of (b). Attractors are indicated by heavier gray edges.

Definition 5.1. Let $s=\left(s_{1}, \ldots, s_{n}\right) \in \mathcal{B}_{\theta}^{n}$. We denote by $\mathcal{I}(s)$ the (multi-)graph with vertex set $V$ and edge set $E(s)$. An edge $e$ is in $E(s)$ if and only if there exists a regular state $s^{\prime}=\left(s_{1}^{\prime}, \ldots, s_{n}^{\prime}\right)$ such that $s_{i}^{\prime}=s_{i}$ for all $i \notin J(s)$ and $e \in E\left(s^{\prime}\right)$, where $E\left(s^{\prime}\right)$ denotes the edge set of the interaction graph $\mathcal{I}\left(s^{\prime}\right)$ in $s^{\prime}$. Again, we call $\mathcal{I}(s)$ the (local) interaction graph in $s$.

Note that the interaction graph in a singular state may have parallel edges. In Fig. 2 (c) we see the local interaction graph in state $(\theta, 0,0)$, which is the union of the graphs $\mathcal{I}((0,0,0))$ and $\mathcal{I}((1,0,0))$ given in (a) and (b).

A singular steady state $s$ yields stability in the dynamical behavior for the components that do not belong to $J(s)$. To make a more precise statement we introduce notation for a specific subgraph of $\mathcal{I}(s)$. By $\mathcal{I}^{\theta}(s)$ we denote the (multi-)graph with vertex set $V^{\theta}(s):=J(s)$ and edge set $E^{\theta}(s):=\{(i, j, \varepsilon) \in E(s) \mid i, j \in J(s)\}$. That is, we only keep the singular components and interactions between them. We call a graph $Z$ component of $\mathcal{I}^{\theta}(s)$, if $Z=\left(V_{Z}, E_{Z}\right)$ is a maximal subgraph of $\mathcal{I}^{\theta}(s)$ such that for every $k, k^{\prime} \in V_{Z}$ exist vertices $k_{1}, \ldots, k_{r} \in V_{Z}$ with $k_{1}=k$, $k_{r}=k^{\prime}$, and $\left(k_{i}, k_{i+1}, \varepsilon\right) \in E^{\theta}(s)$ or $\left(k_{i+1}, k_{i}, \varepsilon\right) \in E^{\theta}(s)$ for some $\varepsilon \in\{+,-\}$ and all $i \in\{1, \ldots, r-1\}$. In Fig. 3 we see for our running example introduced in Fig. 1 the graphs $\mathcal{I}((0, \theta, \theta))$ and $\mathcal{I}^{\theta}((0, \theta, \theta))$ in (a), as well as the graphs $\mathcal{I}((1, \theta, \theta))$ and $\mathcal{I}^{\theta}((1, \theta, \theta))$ in (b). Lastly, let $C$ be a circuit in $\mathcal{I}(s)$ such that all edges of $C$ are in $\mathcal{I}^{\theta}(s)$. Then there exists a component of $\mathcal{I}^{\theta}(s)$ that contains $C$. We denote this component by $J_{C}(s)$. The next lemma shows that the stability of the regular components of a singular steady state is not influenced by value changes in a component $Z$ of $\mathcal{I}^{\theta}(s)$. Moreover, if $\mathcal{I}^{\theta}(s)$ has more than one component, the component dynamics are independent of each other. This property is crucial for the remaining results in this section. The proof of the lemma is an adaptation of a similar, less general statement in [10]. Note that in [10] a different definition of 
$\mathcal{I}^{\theta}(s)$ is used that does not take the effectiveness of interactions in state $s$ into account.

Lemma 5.2. Let $s=\left(s_{1}, \ldots, s_{n}\right)$ be a singular steady state, and let $Z_{1}, \ldots, Z_{m}$ be the components of $\mathcal{I}^{\theta}(s)$. Consider a union $Z$ of arbitrary components $Z_{j}$. Let $\tilde{s}=\left(\tilde{s}_{1}, \ldots, \tilde{s}_{n}\right) \in \mathcal{B}_{\theta}^{n}$ such that $\tilde{s}_{i}=s_{i}$ for all $i \notin Z$. Then $f_{i}^{\theta}(\tilde{s})=f_{i}^{\theta}(s)=s_{i}=\tilde{s}_{i}$ for all $i \notin Z$.

Proof. First, let us consider $i \notin J(s)$. Recall that we denote $[s]:=\left\{s^{\prime} \in \mathcal{B}^{n} \mid\right.$ $s_{j}^{\prime}=s_{j}$ for all $\left.j \notin J(s)\right\}$. Since $s_{j}=\theta$ for all $j \in Z$, we have $J(\tilde{s}) \subseteq J(s)$. Therefore, $[\tilde{s}] \subseteq[s]$. It follows that $K_{i, \min (s)} \leq K_{i, \min (\tilde{s})} \leq K_{i, \max (\tilde{s})} \leq K_{i, \max (s)}$. Since $f_{i}^{\theta}(s)=s_{i}$ is regular, we know $K_{i, \min (s)}=K_{i, \max (s)}=s_{i}$. Thus, $K_{i, \min (\tilde{s})}=$ $K_{i, \max (\tilde{s})}=s_{i}$ and $f_{i}^{\theta}(\tilde{s})=s_{i}=\tilde{s}_{i}$.

Now, let us consider $i \in J(s) \backslash Z$. Then there is no vertex $\alpha_{j}$ in $Z$ that is a predecessor of $\alpha_{i}$ in the local interaction graph $\mathcal{I}(s)$. So there is no interaction from a component in $Z$ to $\alpha_{i}$ that is functional in the part of state space given by $[s]$. Since $[\tilde{s}] \subset[s]$, changes in the values of components in $Z$ do not influence the corresponding value of $f_{i}^{\theta}$. Furthermore, $\tilde{s}_{j}$ coincides with $s_{j}$ for all $j \notin Z$, that is, for all components that might influence the value of $f_{i}^{\theta}(\tilde{s})$. More specifically, since we know that $f_{i}^{\theta}(s)=\theta$, we find $x, y \in[s]$ such that $K_{i, R_{i}(x)}=K_{i, \min (s)}=0$ and $K_{i, R_{i}(x)}=K_{i, \max (s)}=1$. If $z \in\{x, y\}$ does not lie in $[\tilde{s}]$, we can derive a sequence $z=z^{1}, \ldots, z^{k}$ in $[s]$ such that $z^{k} \in[\tilde{s}]$ and every $z^{l}$ differs from $z^{l-1}$ in one component $i_{l} \in Z$ only. Since there are no edges from components in $Z$ to $\alpha_{i}$ in $\mathcal{I}(s)$ we get $K_{i, R_{i}\left(z^{l}\right)}=K_{i, R_{i}(z)}$ for all $l \in\{1, \ldots, k\}$ according to Def. 3 . Thus, we get that $K_{i, \min (s)}=K_{i, \min (\tilde{s})}=0$ and $K_{i, \max (s)}=K_{i, \max (\tilde{s})}=1$, and therefore $f_{i}^{\theta}(\tilde{s})=f_{i}^{\theta}(s)=s_{i}=\tilde{s}_{i}$.

The above lemma shows that the network behavior in $[s]$ is completely governed by the components of $\mathcal{I}^{\theta}(s)$. To give a clear understanding of how to construct the network dynamics from the dynamics derived from the subnetworks we need the following notation.

Let $s$ be a singular steady state and $Z$ a component of $\mathcal{I}^{\theta}(s)$ with $k:=$ card $V_{Z}$. We may assume that $V_{Z}=\left\{\alpha_{l+1}, \ldots, \alpha_{l+k}\right\}$ for some $l \in\{0, \ldots, n-1\}$. Then $Z$ is an interaction graph comprising $k$ vertices. Now, we want to define the dynamics of $Z$ as the projection of the dynamics of $\mathcal{I}$ with respect to $s$. We define a parameter set $K(Z)$ according to Def. 2.2 as the set of all parameters $K_{i, R_{i}^{Z}(z)}^{Z}:=$ $K_{i, R_{i}(\tilde{s})}$ for $z \in \mathcal{B}^{k}$ and $\tilde{s} \in \mathcal{B}^{n}$ with $\tilde{s}_{i}=s_{i}$ for all $i \notin J(s)$ and $\tilde{s}_{i}=z_{i-l}$ for all $i \in Z$. The parameters are well defined since there are no predecessors of vertices in $Z$ in $J(s) \backslash Z$. We set $f^{K(Z)}=f^{Z}: \mathcal{B}^{k} \rightarrow \mathcal{B}^{k}, z \mapsto\left(K_{1, R_{1}^{Z}(z)}^{Z}, \ldots, K_{k, R_{k}^{Z}(z)}^{Z}\right)$. We then have $f^{Z}=\pi^{Z} \circ f^{\theta} \circ \rho^{Z}$, where $\rho^{Z}: \mathcal{B}^{k} \rightarrow \mathcal{B}^{n}$ with $\rho_{i}^{Z}(z)=s_{i}$ for $i \notin Z$ and $\rho_{i}^{Z}(z)=z_{i-l}$ for $i \in Z$, and $\pi^{Z}: \mathcal{B}^{n} \rightarrow \mathcal{B}^{k}$ is the projection on the components of $Z$. Note that $f^{Z}$ yields always regular values, since the singular values in $J(s) \backslash Z$ do not influence the components in $Z$ according to Lem. 5.2. The definitions of parameters and $\mathcal{I}^{\theta}(s)$ ensure that all edges in $Z$ are functional. 
In a next step we can then derive the state transition graph $\mathcal{S}_{Z}=\mathcal{S}_{N^{Z}}$ for the network $N_{Z}=\left(Z, f^{Z}\right)$, the vertex set of which is $\mathcal{B}^{k}$.

We illustrate the definitions by considering our running example in Fig. 1. As shown in Fig. $3(\mathrm{a})$, the graph $\mathcal{I}^{\theta}((0, \theta, \theta))$ has only one component $Z$ consisting of a positive circuit containing $\alpha_{2}$ and $\alpha_{3}$. We derive the parameters $K(Z)$ from those given in Fig. 1 for the global interaction graph. Since $s_{1}=0$, we obtain, according to the above definition, the parameters $K_{2, \emptyset}^{Z}:=K_{2,\left\{e_{12}^{-}, e_{32}^{-}\right\}}=0$ and $K_{2,\left\{e_{32}^{+}\right\}}^{Z}:=K_{2,\left\{e_{12}^{-}, e_{32}^{+}\right\}}=1$. The parameters for $\alpha_{3}$ stay the same, i. e., $K_{3}^{Z}, \omega=$ $K_{3, \omega}$ for $\omega \in\left\{\emptyset,\left\{e_{23}^{+}\right\}\right\}$. The resulting state transition graph $\mathcal{S}_{N}^{Z}$ is also given in Fig. 3 (a).

After calculating the state transition graph for every component of $\mathcal{I}^{\theta}(s)$, we need to find a way to glue them together such that the resulting graph reflects the behavior of our original system in $[s]$.

Definition 5.3. Let $s=\left(s_{1}, \ldots, s_{n}\right)$ be a singular steady state and let $Z_{1}, \ldots, Z_{m}$ be the components of $\mathcal{I}^{\theta}(s)$. W. l. o. g. we may assume that $Z_{1}$ contains the vertices $\alpha_{1}, \ldots, \alpha_{\text {card } Z_{1}}, Z_{2}$ contains the vertices $\alpha_{\text {card } Z_{1}+1}, \ldots, \alpha_{\text {card } Z_{1}+\text { card } Z_{2}}$, etc., and $s_{k}, \ldots, s_{n}$ are all the regular components of $s$ for some $k \in\{1, \ldots, n\}$. We then denote by $\mathcal{S}_{\left(s, Z_{1}, \ldots, Z_{m}\right)}$ the graph with vertex set $V_{\left(s, Z_{1}, \ldots, Z_{m}\right)}:=V_{Z_{1}} \times \cdots \times V_{Z_{m}} \times$ $\left\{\left(s_{k}, \ldots, s_{n}\right)\right\}$ and edge set $E_{\left(s, Z_{1}, \ldots, Z_{m}\right)}$. An edge $s^{1} \rightarrow s^{2}$ belongs to the edge set iff

$$
\pi^{Z_{j}}\left(s^{1}\right)=f^{Z_{j}}\left(\pi^{Z_{j}}\left(s^{1}\right)\right)=\pi^{Z_{j}}\left(s^{2}\right) \text { for all } j \in\{1, \ldots, m\},
$$

or if there exists $j \in\{1, \ldots, m\}$ such that

$$
\pi^{Z_{j}}\left(s^{1}\right) \rightarrow \pi^{Z_{j}}\left(s^{2}\right) \text { is an edge in } \mathcal{S}_{Z_{j}} \text { and } s_{i}^{1}=s_{i}^{2} \text { for all } i \notin V_{Z_{j}} .
$$

We call $\mathcal{S}_{\left(s, Z_{1}, \ldots, Z_{m}\right)}$ the product state transition graph corresponding to $s$.

Furthermore, we denote by $\mathcal{S}_{N}^{[s]}$ the graph with vertex set $[s]$ and edge set $E_{[s]}$. For states $s^{1}$ and $s^{2}$ the edge $s^{1} \rightarrow s^{2}$ is in $E_{[s]}$ iff it is an edge in the state transition graph $\mathcal{S}_{N}$ of $N$.

Note that technically the sets $[s]$ and $V_{\left(s, Z_{1}, \ldots, Z_{m}\right)}$ are not the same, but of course we can identify them with each other and do so to simplify notation. Since $s$ is a singular steady state, we can deduce from Lemma 5.2 that for each $x \in[s]$ we have $x^{\prime} \in[s]$ for all $x^{\prime}$ with $x_{i}^{\prime}=f_{i}(x) \neq x_{i}$ for some $i$ and $x_{j}^{\prime}=x_{j}$ for all $j \neq i$. Thus, there are no edges leaving $[s]$ in the state transition graph $\mathcal{S}_{N}$. The next theorem now tells us that we can reconstruct the behavior of the system $N$ in $[s]$ from the dynamics of the subnetworks $N_{Z_{i}}$.

Theorem 5.4. Let $s=\left(s_{1}, \ldots, s_{n}\right)$ be a singular steady state and let $Z_{1}, \ldots, Z_{m}$ be the components of $\mathcal{I}^{\theta}(s)$. Then $\mathcal{S}_{N}^{[s]}=\mathcal{S}_{\left(s, Z_{1}, \ldots, Z_{m}\right)}$.

Proof. As mentioned above, we identify the sets $[s]$ and $V_{\left(s, Z_{1}, \ldots, Z_{m}\right)}$. Let us remark that we have $\pi^{Z_{j}}\left(f\left(s^{\prime}\right)\right)=\pi^{Z_{j}}\left(f^{\theta}\left(s^{\prime}\right)\right)=\pi^{Z_{j}}\left(f^{\theta}\left(\rho^{Z_{j}}\left(\pi^{Z_{j}}\left(s^{\prime}\right)\right)\right)\right)=f^{Z_{j}}\left(\pi^{Z_{j}}\left(s^{\prime}\right)\right)$ for all $s^{\prime} \in[s]$ and all $j \in\{1, \ldots, m\}$ according to the definition of $\rho^{Z_{j}}, \pi^{Z_{j}}$ and Lemma 5.2. 


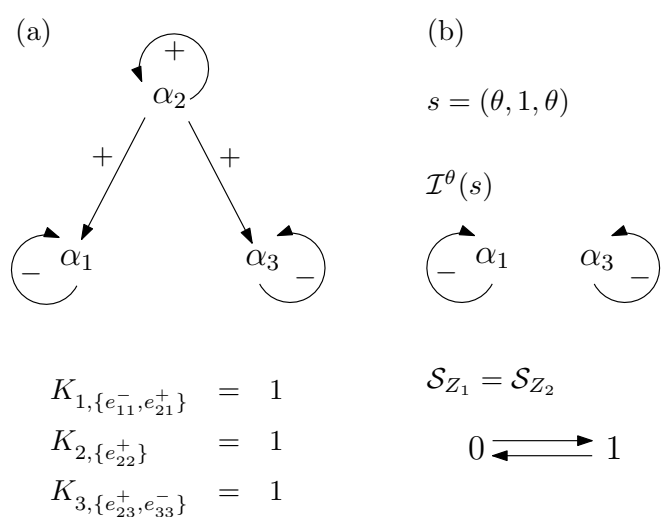

(c)

FIgURE 4. We list only the parameter values greater than zero in (a). The local interaction graph of $s$ in (b) has two components $Z_{1}$ and $Z_{2}$. In (c) the state transition graph of the system in (a).

Let $s^{1}=\left(s_{1}^{1}, \ldots, s_{n}^{1}\right) \rightarrow s^{2}=\left(s_{1}^{2}, \ldots, s_{n}^{2}\right)$ be an edge in $\mathcal{S}_{N}^{[s]}$. According to Def. 2.3, we have either $s^{1}=s^{2}$ is a fixed point of $f$, or $s^{1}$ and $s^{2}$ differ in one component only. If $s^{1}=f\left(s^{1}\right)=s^{2}$, we have $\pi^{Z_{j}}\left(s^{1}\right)=\pi^{Z_{j}}\left(s^{2}\right)$ and $f^{Z_{j}}\left(\pi^{Z_{j}}\left(s^{1}\right)\right)=\pi^{Z_{j}}\left(f\left(s^{1}\right)\right)=\pi^{Z_{j}}\left(s^{1}\right)$ for all $j \in\{1, \ldots, m\}$. Thus, $s^{1} \rightarrow s^{2}$ is an edge in $\mathcal{S}_{\left(s, Z_{1}, \ldots, Z_{m}\right)}$.

Now, let us assume there exists $i \in\{1, \ldots, n\}$ such that $s_{i}^{1} \neq f_{i}\left(s^{1}\right)=s_{i}^{2}$ and $s_{j}^{1}=s_{j}^{2}$ for all $j \neq i$. Then there is $l \in\{1, \ldots, m\}$ such that $i \in Z_{l}$, and $s_{j}^{1}=s_{j}^{2}$ for all $j \notin Z_{l}$. We have to show that $\pi^{Z_{l}}\left(s^{1}\right) \rightarrow \pi^{Z_{l}}\left(s^{2}\right)$ is an edge in $\mathcal{S}_{Z_{l}}$. Let $k^{l}$ be the cardinality of $V_{Z_{l}}$, and let $i_{l} \in\left\{1, \ldots, k^{l}\right\}$ such that $x_{i}=\left(\pi^{Z_{l}}(x)\right)_{i^{l}}$ for all $x \in \mathcal{B}^{n}$, i. e. the $\pi^{Z_{l}}$ projects the $i$-th component of a $n$-vector on the $i^{l}$-th component of a $k$-vector. Then $f_{i^{l}}^{Z_{l}}\left(\pi^{Z_{l}}\left(s^{1}\right)\right)=\left(\pi^{Z_{l}}\left(f\left(s^{1}\right)\right)\right)_{i^{l}}=f_{i}\left(s^{1}\right)=s_{i}^{2}=\left(\pi^{Z_{l}}\left(s^{2}\right)\right)_{i^{l}}$ and $f_{i^{l}}^{Z_{l}}\left(\pi^{Z_{l}}\left(s^{1}\right)\right)=f_{i}\left(s^{1}\right) \neq s_{i}^{1}=\left(\pi^{Z_{l}}\left(s^{1}\right)\right)_{i^{l}}$. Since we know $s_{j}^{1}=s_{j}^{2}$ for all $j \neq i, j \in\{1, \ldots, n\}$, we have $\left(\pi^{Z_{l}}\left(s^{1}\right)\right)_{j^{\prime}}=\left(\pi^{Z_{l}}\left(s^{2}\right)\right)_{j^{\prime}}$ for all $j^{\prime} \neq i^{l}, j^{\prime} \in Z_{l}$. By definition $\pi^{Z_{l}}\left(s^{1}\right) \rightarrow \pi^{Z_{l}}\left(s^{2}\right)$ is an edge in $\mathcal{S}_{Z_{l}}$ and $s^{1} \rightarrow s^{2}$ is an edge in $\mathcal{S}_{\left(s, Z_{1}, \ldots, Z_{m}\right)}$.

Similar straight forward reasoning shows that an edge in $\mathcal{S}_{\left(s, Z_{1}, \ldots, Z_{m}\right)}$ is also an edge in $\mathcal{S}_{N}^{[s]}$.

For the system given in Fig. 4 the state $s=(\theta, 0, \theta)$ is a singular steady state. The components $Z_{1}$ and $Z_{2}$ of $\mathcal{I}^{\theta}(s)$ are the negative loop in $\alpha_{1}$ and the negative loop in $\alpha_{3}$, respectively. The state transition graphs $\mathcal{S}_{Z_{1}}$ and $\mathcal{S}_{Z_{2}}$ coincide and just consist of the cycle $0 \rightarrow 1 \rightarrow 0$. The graph $\mathcal{S}_{\left(s, Z_{1}, Z_{2}\right)}$ is indicated by heavier gray edges in the state transition graph in Fig. 4 (c). Looking at the example in Fig. 1 and the local interaction graphs and corresponding component state transition graphs derived from the singular steady states $s^{1}=(0, \theta, \theta)$ and $s^{2}=(1, \theta, \theta)$ in Fig. 3, we see that the two product state transition graphs derived from them each 
form a component of the state transition graph of the original system. That is, we obtained the whole state transition graph from the subnetworks induced by the singular steady states. The following proposition generalizes this observation. The easy proof is omitted.

Proposition 5.5. If $s^{1}, \ldots, s^{k}$ are singular steady states such that $\mathcal{B}^{n}=\bigcup_{i=1}^{k}\left[s^{i}\right]$, then $\mathcal{S}_{N}=\bigcup_{i=1}^{k} \mathcal{S}_{N}^{\left[s^{i}\right]}$.

In many cases, the above proposition is not very useful. For the system in Fig. 3, for example, there is no singular steady state generating the lower component of the state transition graph. However, we are often not interested in transient states of the system, but rather in the asymptotic behavior. So, we do not necessarily want to reconstruct the complete state transition graph but rather focus on the attractors. According to Prop. 2.5 the graph $\mathcal{S}_{N^{Z}}$ for a component of $\mathcal{I}^{\theta}(s)$ for a singular steady state $s$ contains an attractor. However, it is not a priori clear that attractors of subsystems generate attractors of the complete network. The next theorem shows that this is indeed possible. More precisely, all the attractors in $\mathcal{S}_{N}^{[s]}$ can be constructed from attractors of the subnetworks $Z_{i}$ and vice versa.

Theorem 5.6. Let $s=\left(s_{1}, \ldots, s_{n}\right)$ and $Z_{1}, \ldots, Z_{m}$ be as in Def. 5.3. The attractors of $\mathcal{S}_{N}$ with vertices in $[s]$ are precisely the sets of states that can be represented as $A_{1} \times \cdots \times A_{m} \times\left\{\left(s_{k}, \ldots, s_{n}\right)\right\}$, where for all $i \in\{1, \ldots, m\}$ the set $A_{i}$ denotes an attractor in $\mathcal{S}_{Z_{i}}$.

Proof. For $i \in\{1, \ldots, m\}$ let $A_{i}$ be an attractor in $\mathcal{S}_{Z_{i}}$ and set $A:=A_{1} \times \cdots \times$ $A_{m} \times\left\{\left(s_{k}, \ldots, s_{n}\right)\right\}$. We again interpret $A$ as a subset of $\mathcal{B}^{n}$. First, we show that $A$ is a trap set, i. e., every successor of a state in $A$ is again in $A$. Let $x \in A$ and $x^{\prime}$ be a successor of $x$ in $\mathcal{S}_{N}$, and more precisely in $\mathcal{S}_{N}^{[s]}$. Assume $x \neq x^{\prime}$. Since $\mathcal{S}_{N}^{[s]}=\mathcal{S}_{\left(s, Z_{1}, \ldots, Z_{m}\right)}$, we infer from Def. 5.3 that there exists $j \in\{1, \ldots, m\}$ such that $\pi^{Z_{j}}(x) \rightarrow \pi^{Z_{j}}\left(x^{\prime}\right)$ is an edge in $\mathcal{S}_{Z_{j}}$. Since $\pi^{Z_{j}}(x) \in A_{j}$ and since $A_{j}$ is an attractor, we have $\pi^{Z_{j}}\left(x^{\prime}\right) \in A_{j}$. Since $x$ and $x^{\prime}$ only differ in one component, we have $x^{\prime} \in A$.

Now, we have to show that there is a path from $x$ to $x^{\prime}$ in $\mathcal{S}_{N}$ for all distinct $x, x^{\prime} \in A$. First, we note that if there is an edge from state $z$ to state $z^{\prime}, z \neq z^{\prime}$, in $\mathcal{S}_{Z_{l}}, l \in\{1, \ldots, m\}$, then there is an edge from $x$ to $x^{\prime}$ in $\mathcal{S}_{N}$ for all states $x, x^{\prime} \in A$ satisfying $\pi^{Z_{l}}(x)=z, \pi^{Z_{l}}\left(x^{\prime}\right)=z^{\prime}$, and $x_{j}=x_{j}^{\prime}$ for all $j \notin Z_{l}$, according to the definition of edges in $\mathcal{S}_{\left(s, Z_{1}, \ldots, Z_{m}\right)}=\mathcal{S}_{N}^{[s]}$.

Let $x, x^{\prime} \in A$. We set $x_{i}^{1}:=x_{i}$ for all $i \notin Z_{1}$ and $x_{i}^{1}:=x_{i}^{\prime}$ for all $i \in Z_{1}$. For $l \in\{2, \ldots, m\}$ we set $x_{i}^{l}:=x_{i}^{l-1}$ for all $i \notin Z_{l}$ and $x_{i}^{l}:=x_{i}^{\prime}$ for all $i \in Z_{l}$. Then there exists a path in $\mathcal{S}_{N^{Z_{1}}}$ from $\pi^{Z_{1}}(x)$ to $\pi^{Z^{1}}\left(x^{1}\right)$, since $A_{1}$ is an attractor. As seen above, we then can find a path $\gamma_{1}$ from $x$ to $x^{1}$ in $\mathcal{S}_{N}$ such that $\tilde{x}_{j}=x_{j}$ for every state $\tilde{x} \in \gamma_{1}$ and every $j \notin Z_{1}$. In the same fashion we find a path $\gamma_{2}$ from $x^{1}$ to $x^{2}$ in $\mathcal{S}_{n}$ such that $\tilde{x}_{j}=x_{j}^{1}$ for all $\tilde{x} \in \gamma_{2}$ and $j \notin Z_{2}$. We continue the procedure for $Z_{3}, \ldots, Z_{m}$. Since $x^{m}=x^{\prime}$ per definition, combining the paths $\gamma_{i}$ in 
the order of their indices yields a path from $x$ to $x^{\prime}$ in $\mathcal{S}_{N}$. It follows that $A$ is an attractor in $\mathcal{S}_{N}$.

Now, let $A$ be in arbitrary attractor in $\mathcal{S}_{N}$ with vertices in $[s]$ and consider the set $A_{j}=\pi^{Z_{j}}(A)$ for $j \in\{1, \ldots, m\}$. $A_{j}$ has to be a trap set in $\mathcal{S}_{Z_{j}}$ since edges leaving $A_{j}$ would generate edges leaving $A$ in $\mathcal{S}_{\left(s, Z_{1}, \ldots, Z_{m}\right)}=\mathcal{S}_{N}^{[s]}$ according to Def. 5.3. Let $x, x^{\prime} \in A_{j}$. Then there exist states $y, y^{\prime} \in A$ such that $x=\pi^{Z_{j}}(y)$ and $x=\pi^{Z_{j}}(y)$. Since $A$ is an attractor, there is a path $\gamma$ from $y$ to $y^{\prime}$ in $\mathcal{S}_{N}$, and more precisely in $\mathcal{S}_{N}^{[s]}$, since $[s]$ is a trap set. It is easy to verify that we obtain a path from $x$ to $x^{\prime}$ in $\mathcal{S}_{N_{j}}$ by projecting the vertices of $\gamma$ onto $Z_{j}$ and eliminating all but one of consecutive identical vectors in the resulting sequence of states. The existence of edges in $\mathcal{S}_{N_{j}}$ between the remaining vertices is again guaranteed by Def. 5.3.

Let us use the example in Fig. 1 to illustrate the theorem. In Fig. 3 (a), we see the state transition graph $\mathcal{S}_{N}^{Z}$ for the single component $Z$ of $\mathcal{I}^{\theta}(s)$. It contains the attractors $\{(0,0)\}$ and $\{(1,1)\}$. It follows from Theorem 5.6 that the sets $\{(0,0,0)\}$ and $\{(0,1,1)\}$ are attractors in $\mathcal{S}_{N}$. Similarly, we derive a state transition graph from $\mathcal{I}^{\theta}((1, \theta, \theta))$ which consists of a negative circuit. The state transition graph is shown in Fig. 3 (b) and contains only one attractor, the set $\{(0,0),(1,0),(1,1),(0,1)\}$, which has cardinality greater than one. Thus, we find an attractor $\{(1,0,0),(1,1,0),(1,1,1),(1,0,1)\}$ in $\mathcal{S}_{N}$. The state transition graph $\mathcal{S}_{N}$ is given in Fig. 1 with the attractors emphasized.

The number and size of attractors are important characteristics of bioregulatory networks. There are many results that link these dynamical characteristics to properties of the network structure. In [3] it is shown that isolated circuits always display a characteristic behavior depending on their sign. A positive circuit gives rise to two attractors, more precisely two steady states, a negative circuit results in a cyclic attractor, i. e., an attractor with cardinality greater than one. The situation is much more difficult to analyze if there are many circuits in $\mathcal{I}$, possibly even intertwined. Thomas conjectured in 1981 that the existence of a positive resp. negative circuit in the interaction graph is a necessary condition for the existence of two attractors resp. a cyclic attractor in the state transition graph. The conjectures haven been proven in different settings (see e.g. [12], [4] and [6]). For regulatory networks without context sensitivity, we formulated in [10] a sufficient condition for circuits to display their characteristic behavior using singular steady states. The proof in [10] can be easily adapted to show the next statement.

Lemma 5.7. Let $I$ be an interaction graph that contains only one circuit $C$. If $C$ is a positive circuit, then $f$ has two fixed points. If $C$ is negative, then there exists an attractor with cardinality greater than one in the state transition graph.

We make some short remarks on the proof. Recall our assumption that every vertex in $\mathcal{I}$ has a predecessor. Since every edge is functional, the state $(\theta, \ldots, \theta)$ is steady. In [10], it is shown that $\mathcal{I}$ then has a particular structure. It consists of the circuit $C$ with directed acyclic graphs coming out of vertices of $C$. This structure 


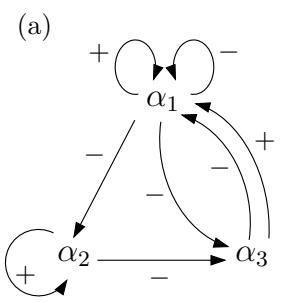

(b)

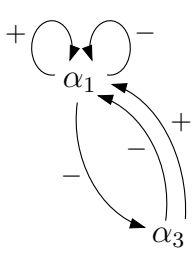

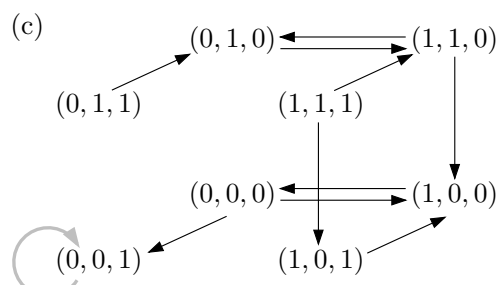

FiguRE 5. We choose the parameters for the interaction graph in (a) as $K_{1,\left\{e_{11}^{+}, e_{31}^{+}\right\}}=K_{1,\left\{e_{11}^{-}, e_{31}^{-}\right\}}=K_{2,\left\{e_{12}^{-}, e_{22}^{+}\right\}}=K_{3,\left\{e_{13}^{-}, e_{23}^{-}\right\}}=1$ and set all other parameters 0 . In (b) the graph $\mathcal{I}^{\theta}(s)$ for the singular steady state $s=(\theta, 0, \theta)$. In (c) the corresponding state transition graph.

allows us to explicitly specify values for the vertices of $C$ that remain fix under $f^{\theta}$ in the case of $C$ being positive, or behave like a trap cycle, if $C$ is negative. From this core behavior we can then infer the behavior of the whole graph. Here, we also have to consider that there may be parallel edges outside the circuit $C$. However, the proof method is still valid. The necessary technical adaptations to the proofs in [10] correspond to those made in the proof of Lemma 5.2.

The above lemma together with Theorem 5.6 leads to the following theorem. Recall that we denote the component of $\mathcal{I}^{\theta}(s)$ containing some circuit $C$ by $J_{C}(s)$.

Theorem 5.8. Let $C$ be a circuit in $\mathcal{I}$ and $s$ a singular steady state characteristic of $C$. Assume that $C$ is the only circuit in the component $J_{C}(s)$ of $\mathcal{I}^{\theta}(s)$. If $C$ is a positive circuit, then $f^{\theta}$ has at least three fixed points and $\mathcal{S}_{N}$ contains at least two attractors. If $C$ is negative, there is an attractor in $\mathcal{S}_{N}$ with cardinality greater than one.

Proof. We may assume that $J_{C}(s)$ comprises the vertices $\alpha_{1}, \ldots, \alpha_{r}$ for some $r \in$ $\{1, \ldots, N\}$. Let at first $C$ be positive. Then $f^{J_{C}(s)}$ has two fixed points $x, x^{\prime} \in \mathcal{B}^{r}$ according to Lemma 5.7. We define states $s^{1}$ and $s^{2}$ in $\mathcal{B}_{\theta}^{n}$ by $s_{i}^{1}:=s_{i}^{2}:=s_{i}$ for all $i \notin J_{C}(s), s_{i}^{1}:=x_{i}$ and $s_{i}^{2}:=x_{i}^{\prime}$ for all $i \in\{1, \ldots, r\}$. From Lemma 5.2 follows that the states $s^{1}$ and $s^{2}$ are steady states. Thus $f^{\theta}$ has three fixed points, since $s$ is distinct from $s^{1}$ and $s^{2}$. According to Theorem 5.6 we find attractors $A_{1}$ and $A_{2}$ in $\mathcal{S}_{N}$ such that $\pi^{J_{C}(s)}\left(A_{1}\right)=\left\{s^{1}\right\}$ and $\pi^{J_{C}(s)}\left(A_{2}\right)=\left\{s^{2}\right\}$.

If $C$ is negative, we find an attractor $A^{\prime}$ in the state transition graph of the component graph $J_{C}(s)$ with card $A^{\prime}>1$. Theorem 5.6 yields an attractor $A$ in $\mathcal{S}_{N}$ with $\pi^{J_{C}(s)}(A)=A^{\prime}$. Thus cardinality of $A$ is also greater than one.

Theorem 5.8 is a stronger result than the one obtained in [10], even for networks without context sensitivity. The use of local interaction graphs allows for a more refined picture of the dynamics possible in restricted parts of the state space. 
Our running example from Fig. 1 together with Fig. 3 illustrates the theorem. Figure 5 shows that the statement does not hold, if the circuit $C$ is not the only circuit in $J_{C}(s)$. The state $(\theta, 0, \theta)$ is steady for the bioregulatory network derived from the interaction graph in (a) and the parameters specified in the caption. There are four circuits in $\mathcal{I}^{\theta}((\theta, 0, \theta))$, two negative and two positive circuits. However, the state transition graph contains only one attractor, namely the set $\{(0,0,1)\}$, as is shown in (c). Neither the behavior characteristic for positive circuits nor that characteristic for negative circuits is displayed. Further examples can be found in [10]. However, a system may display the behavior characteristic for a circuit of a given sign, although there is no singular steady $s$ such that the circuit is the only one in the corresponding component of $\mathcal{I}^{\theta}(s)$. The condition is not necessary, as illustrated by an example given in [10], Fig. 4 .

\section{Networks with Input Layer}

In the preceding section we gave several results on how to derive information on the behavior of a complex network by looking at suitable subnetworks, which in turn we derived from singular steady states. However, in most cases the results do not yield a complete analysis of the system's dynamics. To obtain more comprehensive results we are faced with two difficulties. First, we need to make sure that our in nature local analysis covers all parts of state space containing asymptotically stable behavior. This is a difficult task, since we in general do not know anything about the network dynamics a priori. This directly relates to the second difficulty. Since we use singular steady states to deconstruct the network, we need a method to find singular steady states, and, coming back to the first problem, in particular to identify a set of singular steady states such that the generated subnetworks hold sufficient information to characterize the global dynamics of the whole system.

In this section we introduce a class of networks for which we can solve the problems described above. In the following, we only consider interaction graphs the underlying undirected graph of which is connected. For the general case, we just consider the components separately.

Definition 6.1. We call $N$ a network with input layer, if there exists a vertex $\alpha_{i}$ with a positive edge to itself and no other incoming edges. A vertex satisfying this condition is called input vertex.

In the following we assume that $N$ is a network with input layer. Without loss of generality we assume that $\alpha_{1}, \ldots, \alpha_{k}$ are the input vertices of $N$. In Fig. 6 we see a network with input layer. The components $\alpha_{1}$ and $\alpha_{2}$ are the input vertices.

Networks with input layer often play an important role in biological systems. When modeling signal transduction networks, for example, we can model receptors as input vertices. Different input values then represent different signals reaching the receptors, and we want to understand how the system reacts to such signals.

If a system has an input layer, then we can immediately make some observation about the network dynamics. The coordinate function $f_{i}$ governing the 
behavior of the input vertex $\alpha_{i}$ solely depends on the value of $\alpha_{i}$. Since $\alpha_{i}$ influences itself via a positive edge, we have $f_{i}(x)=x_{i}$ for all $x \in \mathcal{B}^{n}$ according to condition (3.1). That is, the input vertex values always stay fixed and therefore the state transition graph consists of $2^{k}$ components. In particular, we know that each attractor of the system is contained in one of the components. This proves the statement of the following lemma.

Lemma 6.2. Let $A$ be an attractor in $\mathcal{S}_{N}$. Then $a_{i}=a_{i}^{\prime}$ for all $a, a^{\prime} \in A$ and $i \in\{1, \ldots, k\}$.

The specific structure of a network with input layer allows us to find singular, or possibly even regular, steady states in a simple way. Recall that we denote the set of singular components of a state $s$ by $J(s)$.

Lemma 6.3. Let $x_{1}, \ldots, x_{k} \in \mathcal{B}$. Define $x^{0} \in \mathcal{B}_{\theta}^{n}$ by $x_{i}^{0}:=x_{i}$ for all $i \in\{1, \ldots, k\}$ and $x_{i}^{0}:=\theta$ for $i>k$. Then the sequence $\left(x^{l}\right)_{l \in \mathbb{N}}$ where $x^{l}:=f^{\theta}\left(x^{l-1}\right)$ converges to a fixed point $s$ of $f^{\theta}$. Moreover, if we set $x_{i}^{0}:=x_{i}$ for all $i \in\{1, \ldots, k\}$ and arbitrarily choose $x_{i}^{0} \in \mathcal{B}$ for $i>k$ and define the sequence $\left(x^{l}\right)_{l \in \mathbb{N}}$ as above, then there exists $l \in \mathbb{N}$ such that $x_{i}^{m}=s_{i}$ for all $i \in\{1, \ldots, n\} \backslash J(s)$ and $m \geq l$.

Proof. First, we show by induction that if $x_{i}^{l}$ is a regular value for some $l \in \mathbb{N}$ and some $i \in\{1, \ldots, n\}$, then $x_{i}^{m}=x_{i}^{l}$ for all $m \geq l$. The only regular values of $x^{0}$ correspond to the input vertices $\alpha_{1}, \ldots, \alpha_{k}$, and we have $x_{i}^{m}=x_{i}^{0}$ for all $m \in \mathbb{N}$ and $i \in\{1, \ldots, k\}$. Let $l \in \mathbb{N}$. We assume that if $x_{i}^{l} \in\{0,1\}$ for some $i \in\{1, \ldots, n\}$, then $x_{i}^{m}=x_{i}^{l}$ for all $m \geq l$.

Let $i \in\{1, \ldots, n\}$ such that $x_{i}^{l+1} \in\{0,1\}$. If $i \notin J\left(x^{l}\right)$, our assumption yields $x_{i}^{m}=x_{i}^{l}=x_{i}^{l+1}$ for all $m \geq l+1$. Let $i \in J\left(x^{l}\right)$. Since $x_{i}^{l+1}=f_{i}^{\theta}\left(x^{l}\right)$ is a regular value, we have $f_{i}^{\theta}\left(x^{l}\right)=K_{i, \min \left(x^{l}\right)}=K_{i, \max \left(x^{l}\right)}$. Since $x^{l}$ and $x^{m}$ coincide in all regular values of $x^{l}$ for all $m \geq l$, we have $x^{m} \in\left[x^{l}\right]$ for all $m \geq l$. It follows from the definition of $K_{i, \min \left(x^{m}\right)}$ and $K_{i, \max \left(x^{m}\right)}$ that $K_{i, \min \left(x^{l}\right)} \leq K_{i, \min \left(x^{m}\right)} \leq$ $K_{i, \max \left(x^{m}\right)} \leq K_{i, \max \left(x^{l}\right)}$, and thus $K_{i, \min \left(x^{m}\right)}=K_{i, \max \left(x^{m}\right)}$ for all $m \geq l$. We obtain $x_{i}^{m+1}=f^{\theta}\left(x^{m}\right)=K_{i, \min \left(x^{m}\right)}=K_{i, \min \left(x^{l}\right)}=x_{i}^{l+1}$ for all $m \geq l$, which proves our statement. Since we obtain the sequence $\left(x^{l}\right)_{l \in \mathbb{N}}$ by iteration of $f^{\theta}$ and since $\mathcal{B}_{\theta}^{n}$ is finite, the sequence converges to a regular or singular fixed point of $f^{\theta}$.

Now set $\tilde{x}_{i}^{0}:=x_{i}^{0}$ for $i \in\{1, \ldots, k\}$, choose $\tilde{x}_{i} \in \mathcal{B}$ for $i>k$ and define $\tilde{x}^{l+1}:=f\left(\tilde{x}^{l}\right)$ for $l \in \mathbb{N}$. Then, we have $\tilde{x}^{0} \in\left[x^{0}\right]$ and thus it follows with the same reasoning as above that $\tilde{x}_{i}^{1}=x_{i}^{1}$ for all $i \notin J\left(x^{1}\right)$. Again, we have $\tilde{x}^{1} \in\left[x^{1}\right]$ and it follows $\tilde{x}_{i}^{2}=x_{i}^{2}$ for all $i \notin J\left(x^{2}\right)$. Repeating the argument until we reach the fixed point $s$ generated by $\left(x^{l}\right)_{l \in \mathbb{N}}$, we find $l \in \mathbb{N}$ such that $\tilde{x}_{i}^{m}=s_{i}$ for all $m \geq l$ and $i \notin J(s)$.

We call the resulting fixed point in the preceding lemma the fixed point derived from the input values $x_{1}, \ldots, x_{k}$. The above lemma provides a method to translate combinations of input values into singular or regular steady states. It turns out that if we calculate the fixed points derived from all possible combinations of input values, then we can completely describe the asymptotic behavior of 
Heike Siebert

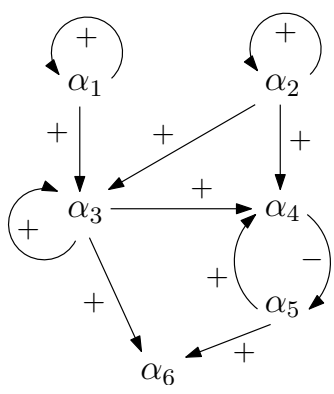

\begin{tabular}{c|c|l}
$\left(s_{1}, s_{2}\right)$ & $f(s)=s$ & attractors \\
\hline$(0,0)$ & $(0,0, \theta, 0,0, \theta)$ & $\{(0,0,0,0,0,0)\},\{(0,0,1,0,0,1)\}$ \\
$(1,0)$ & $(1,0,1,0,0,1)$ & $\{(1,0,1,0,0,1)\}$ \\
$(1,1)$ & $(1,1,1, \theta, \theta, 1)$ & $\{(1,1,1, x, y, 1) \mid x, y \in\{0,1\}\}$ \\
$(0,1)$ & $(0,1,1, \theta, \theta, 1)$ & $\{(0,1,1, x, y, 1) \mid x, y \in\{0,1\}\}$
\end{tabular}

Figure 6. We choose the parameters for the given interaction graph such that the inputs to $\alpha_{3}$ and $\alpha_{6}$ operate via an OR gate, while inputs to $\alpha_{4}$ are processed via an AND gate. The left column in the table lists the different input values for the network, the middle column the derived singular steady states, and the right column the resulting attractors.

the network by considering resulting regular steady states and the subnetworks obtained from the resulting singular steady states.

Theorem 6.4. Let $A$ be an attractor of $N$. Then there exist input values $x_{1}, \ldots, x_{k} \in$ $\mathcal{B}$ such that either $A=\{s\}$ or we can construct $A$ from s as shown in Theorem 5.6, where $s=\left(s_{1}, \ldots, s_{n}\right)$ is the fixed point derived from $x_{1}, \ldots, x_{k}$.

Proof. Let $a^{\prime} \in A$ and set $x_{i}:=a_{i}^{\prime}$ for all $i \in\{1, \ldots, k\}$. Let $s$ be the fixed point derived from $x_{1}, \ldots, x_{k}$. According to Lemma 6.2, the first $k$ components remain fix in $A$, and thus $a_{i}=x_{i}=s_{i}$ for all $i \in\{1, \ldots, k\}$. Since $A$ is a trap set, we can deduce from the second statement in Lemma 6.3 that $a_{i}=s_{i}$ for all $a \in A$ and $i \in\{1, \ldots, n\} \backslash J(s)$, since those values remain fixed under $f$ after sufficiently many iteration steps. It follows that $A \subseteq[s]$. If $s$ is a singular steady state, then we can construct $A$ from the attractors of the subnetworks derived from $s$ according to Theorem 5.6. Otherwise, we have $J(s)=\emptyset$ and thus $A$ consists of the regular fixed point $S$.

We illustrate the theorem with the system given in Fig. 6. The table on the left of the figure shows in the left column the possible combinations of input values, in the middle column the fixed point derived from those values, and in the right column the resulting attractors of the system. The input $\left(\alpha_{1}, \alpha_{2}\right)=$ $(0,0)$ yields the singular steady state $(0,0, \theta, 0,0, \theta)$. The subnetwork governing the behavior of the system in the corresponding part of state space consists of the positive loop in $\alpha_{3}$ and the edge from $\alpha_{3}$ to $\alpha_{6}$. This subsystems has two fixed points $(0,0)$ and $(1,1)$. Thus, we obtain two steady states of the original system, namely $(0,0,0,0,0,0)$ and $(0,0,1,0,0,1)$. The input $(1,0)$ directly leads to the regular steady state $(1,0,1,0,0,1)$. Both input vectors $(1,1)$ and $(0,1)$ generate a singular steady state the local interaction graph of which consist of the negative 
circuit between $\alpha_{4}$ and $\alpha_{5}$. This subsystem has only one attractor consisting of the states $(0,0),(0,1),(1,1)$ and $(1,0)$. So we obtain a cyclic attractor of the original system for each of the two input vectors. According to Theorem 6.4 the five listed attractors are all the attractors of the system, which is easily confirmed when calculating the whole state transition graph.

\section{Conclusion}

The focus of this paper is to obtain information about the dynamics of complex regulatory networks by analyzing subsystems of the network, thereby reducing the complexity of the problem. To identify suitable subnetworks we use the notion of singular steady state, which we introduced in [10] in a more restricted framework. In addition we employ the idea of local interaction graphs in order to obtain a refined understanding of what interactions in the network structure measurably influence the behavior of the system in any given part of state space. We show in Sect. 5 that the information about the network behavior encoded in a given singular steady state together with the refined structural representation of a corresponding interaction graph allows us to derive the dynamics of the whole network, at least in part of state space, and show how to construct attractors of the network from attractors of subnetworks. This approach also yields a deeper understanding of the relation between structural and dynamical network characteristics. We obtain a result linking the existence of circuits in the interaction graph to the existence of multiple attractors resp. an attractor with cardinality greater than one, which generalizes and refines a corresponding statement in [10]. Feedback circuits are known to be an important building block, or so-called network motif, for bioregulatory networks. Our result gives sufficient conditions for them to imprint the behavior characteristic for them in isolation on a complex network containing such circuits. Other important structural network motifs have been characterized, and in this context application of our methods may help to analyze their behavior if embedded in a complex network.

In future work, we also plan to focus more on the application side. Sect. 6 introduces a solid starting point for this endeavor. In particular, the analysis of signal transduction networks is of interest for further studies. However, when modeling biological systems, Boolean networks, although often yielding a fruitful first model, can only provide a very course description. Multi-valued models allow for finer representation, still preserving the advantages of discrete modeling. Generalizing the results presented in this paper to the framework of multi-valued discrete networks thus is another priority for future work. 


\section{References}

[1] G. Bernot, J.-P. Comet, A. Richard, and J. Guespin. Application of formal methods to biological regulatory networks: extending Thomas' asynchronous logical approach with temporal logic. J. Theor. Biol., 229:339-347, 2004.

[2] C. Chaouiya, É. Remy, B. Mossé, and D. Thieffry. Qualitative analysis of regulatory graphs: a computational tool based on a discrete formal framework. In First Multidisciplinary International Symposium on Positive Systems: Theory and Applications, POSTA 2003, volume 294 of LNCIS, pages 119-126. Springer, 2003.

[3] É. Remy, B. Mossé, C. Chaouiya, and D. Thieffry. A description of dynamical graphs associated to elementary regulatory circuits. Bioinform., 19:172-178, 2003.

[4] É. Remy and P. Ruet. On differentiation and homeostatic behaviours of Boolean dynamical systems. In Transactions on Computational Systems Biology VIII, volume 4780 of $L N C S$, pages 92-101. Springer, 2007.

[5] É. Remy, P. Ruet, and D. Thieffry. Graphic requirements for multistability and attractive cycles in a boolean dynamical framework. Prépublication, 2005.

[6] A. Richard and J.-P. Comet. Necessary conditions for multistationarity in discrete dynamical systems. Rapport de Recherche, 2005.

[7] A. Richard, J.-P. Comet, and G.Bernot. R. Thomas' modeling of biological regulatory networks: introduction of singular states in the qualitative dynamics. Fundamenta Informaticae, 65:373-392, 2005.

[8] F. Robert. Discrete Iterations: A Metric Study, volume 6 of Springer Series in Computational Mathematics. Springer, 1986.

[9] H. Siebert. Local structure and behavior of boolean bioregulatory networks. In Algebraic Biology, AB 2008, Castle of Hagenberg, Austria, volume 5147 of LNCS, pages 185-199. Springer, 2008.

[10] H. Siebert and A. Bockmayr. Relating attractors and singular steady states in the logical analysis of bioregulatory networks. In Algebraic Biology, AB 2007, Castle of Hagenberg, Austria, volume 4545 of LNCS, pages 36-50. Springer, 2007.

[11] E. H. Snoussi and R. Thomas. Logical identification of all steady states: the concept of feedback loop characteristic states. Bull. Math. Biol., 55:973-991, 1993.

[12] C. Soulé. Graphical requirements for multistationarity. ComPlexUs, 1:123-133, 2003.

[13] R. Thomas and R. d'Ari. Biological Feedback. CRC Press, 1990.

[14] R. Thomas and M. Kaufman. Multistationarity, the basis of cell differentiation and memory. II. Logical analysis of regulatory networks in terms of feedback circuits. Chaos, 11:180-195, 2001.

Heike Siebert

DFG Research Center MAThEON

Freie Universität Berlin

Arnimallee 6

D-14195 Berlin

Germany

e-mail: siebert@mi.fu-berlin.de 